\title{
Fitness and microbial networks of the common wasp, Vespula vulgaris (Hymenoptera: Vespidae), in its native and introduced ranges
}

M O N I C A. M. GRUBER, ${ }^{1,2 *}$ OL I V E R Q U I N N, ${ }^{1 *}$ J A M E S W. B A T Y, ${ }^{1}$ J A N A D O B E L M A N N, ${ }^{1} \mathrm{JOH} \mathrm{H} \mathrm{H} \mathrm{A} \mathrm{Y} \mathrm{W} \mathrm{O} \mathrm{O} \mathrm{D,}^{3}$

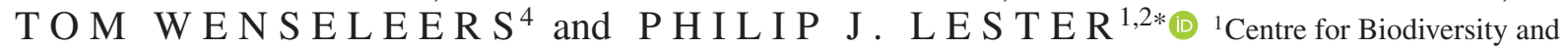
Restoration Ecology, School of Biological Sciences, Victoria University of Wellington, Wellington, New Zealand, ${ }^{2}$ Pacific Biosecurity, Victoria Link Limited, Victoria University of Wellington, Wellington, New Zealand, ${ }^{3}$ School of Mathematics and Statistics, Victoria University of Wellington, Wellington, New Zealand and ${ }^{4}$ Department of Biology, Laboratory of Socioecology and Social Evolution, Leuven, Belgium

\begin{abstract}
Variation in microbial communities between populations is increasingly hypothesised to affect animal fitness and performance, including for invasive species. Pathogenic species may be lost during the introduction process, enhancing invader fitness and abundance.

2. This study assessed fitness, immune gene expression, and microbial network complexity of invasive common wasps, Vespula vulgaris. Microbial networks were assayed using $16 \mathrm{~S}$ and $18 \mathrm{~S}$ sequencing and gene expression arrays in the native (Belgium) and introduced range (New Zealand). The immune gene expression of the wasp Down syndrome cell adhesion molecule (Dscam) gene homologue was examined. Dscam expression can be induced by viruses, Gram-positive and Gram-negative bacteria, and parasites.

3. Individual nest fitness was higher in the native range of Belgium than in the introduced New Zealand range. Microbial communities of wasps in the introduced range were more diverse with more complex networks, although some microorganisms were range-specific. Microbial networks in the introduced range showed higher clustering coefficients, number of connected paths, network centralisation, number of neighbours and network density.

4. Larvae, workers, virgin and foundress queens had higher expression of Dscam in the New Zealand samples. These immune gene expression patterns were associated with higher pathogen pressure and lower relative fitness.

5. Epidemiological theory predicts that a high density of pathogen and microbial hosts should result in a high rate of disease infection, prevalence, and highly connected microbial networks. The results of this study support these predictions. Wasps displayed lower relative fitness and more highly connected microbial networks in New Zealand than in Belgium.
\end{abstract}

Key words. Bacteria, fungi, invasive species, pathogens, social wasps, viruses.

Correspondence: Philip J. Lester, School of Biological Sciences, Victoria University of Wellington, P.O. Box 600, Wellington 6140, New Zealand. E-mail: Phil.Lester@vuw.ac.nz

*These authors contributed equally to this study. 


\section{Introduction}

There is an increasing awareness that microbial communities can affect animal fitness and performance. Microbial communities in the gut, for example, are involved in much more than just nutrition. These communities can be vital for host immunity and shape life-history traits or animal behaviour (Shapira, 2016). A developing paradigm suggests that individuals cannot be considered truly independent of the microbial communities they host, with even minor perturbations to hosts and their microbial communities having long-term health and fitness impacts (McFall-Ngai et al., 2013). A substantial perturbation is a biological introduction event, wherein a population is moved to a new area, leading to the formation of new microbial communities in the host (e.g. Lester et al., 2017b).

The common wasp, Vespula vulgaris, is listed in a selection of the world's worst invasive species (Lowe et al., 2000). This omnivorous wasp is native to Eurasia and has had multiple introductions to countries such as Argentina, Australia and New Zealand (Lester et al., 2014). In their introduced range, the ecological impacts of common wasps are density-dependent. For example, in beech forests of New Zealand, large populations of common wasps are predators that decimate insect communities and compete with forest birds (Lester \& Beggs, 2019). In these forests they reach the highest known nest densities, with up to 40 nests ha ${ }^{-1}$ (Lester et al., 2017a). In comparison, a 32-year annual survey from the native range of England showed an average of just 0.45 common wasp nests ha ${ }^{-1}$ (range $=0.35-1.78$ ) (Archer $\&$ Halstead, 2014). These wasps thus appear to 'perform better' in their introduced range (Parker et al., 2013).

The search for biological control agents for introduced populations of this wasp has detected a number of potential pathogens commonly found in honey bees. These include Deformed wing virus, Kashmir bee virus, Nosema, Aspergillus, as well as potential commensals, such as Bifidobacterium, Vagococcus, and Streptococcus (Glare et al., 1996; Rose et al., 1999; Evison et al., 2012). These microbial pathogens have been hypothesised to play a substantial role in honey bee health and to contribute to their population collapse (Bromenshenk et al., 2010; Evans \& Schwarz, 2011; Cornman et al., 2012). Mutualistic or commensal microbial symbionts can also enhance nutrition and immune defence of their social insect hosts, either facultatively or obligately (Kaltenpoth \& Engl, 2014). Differences in the abundance and diversity of pathogens and perhaps entire microbial communities within wasps might play a part in the variation in performance and abundance of common wasps.

Here, we first examine the hypothesis that the relative fitness of wasps will be higher in their introduced range in New Zealand compared with that in their native Belgium. We then examine the microbial communities in the two regions. Due to the introduction bottleneck (Lester et al., 2014) and perhaps an escape from natural enemies (Torchin et al., 2003), we expected that the microbial interaction network associated with wasps would be considerably more complex in native Belgium than in the examined introduced populations in New Zealand. We hypothesised that the variation in wasp fitness, and the higher performance of wasps in the introduced range of New Zealand, would be associated with differences in microbial community diversity. Finally, we examined the relative expression of the wasp Down syndrome cell adhesion molecule (Dscam) gene homologue. Splicing of this gene results in thousands of isoforms (Schmucker et al., 2000; Armitage et al., 2015). Ng et al. (2014) reviewed the role of Dscam in both the general innate immunity and the pathogen-specific immune response of invertebrates. They found evidence that Dscam expression can be modified by viruses, Gram-positive and Gram-negative bacteria, pathogen association molecular patterns, and parasites. Dscam has previously been shown to be significantly up-regulated in virus- and microsporidian-infected honey bees (Flenniken \& Andino, 2013; Schwarz \& Evans, 2013; Brutscher et al., 2015). Under a scenario of enemy release (Keane \& Crawley, 2002; Torchin et al., 2003), we would expect to see lower Dscam expression in the introduced range of New Zealand.

\section{Materials and methods}

\section{Fitness of wasps in the native and introduced ranges}

Common wasps were introduced and became abundant in New Zealand in the 1970s, and appear genetically similar to those in Belgium, the U.K. or other areas of western Europe, which are all probable source populations (Lester et al., 2014). We collected wasp nests in late autumn for 3 years between 2014 and 2016 in part of both the native range (Belgium) and an introduced country (New Zealand) (Appendix S1) in the period they were producing new sexuals. We attempted to sample additional sites in the native range of England and the introduced a range from Argentina, but a lack of available wasp nests in England in the year of sampling and volcanic activity in Argentina restricted our sampling in these locations.

The annual cycle of a common wasp nest begins with a single inseminated queen initiating a new nest in spring. She produces sterile worker wasps from spring until autumn. New virgin queens (gynes) and males are produced in autumn. The number of these new queens produced is correlated with worker numbers (Spradbery, 1971), which have the role of provisioning and tending the virgin queens. The autumn nests were located by observing foraging workers. Each nest was excavated and transferred into a sealed bucket, which was then chilled at $-20{ }^{\circ} \mathrm{C}$ for $c .2 \mathrm{~h}$. After chilling, all wasps were alive but able to be handled. A sample of $c .30$ individuals of each life stage of live fourth- or fifth-instar larvae, adult workers, and virgin queens was randomly collected from every nest and snap-frozen in liquid nitrogen. Samples were then stored at $-80^{\circ} \mathrm{C}$ before RNA extraction. For the $16 \mathrm{~S}$ and $18 \mathrm{~S}$ amplicon sequencing, a single nest was sampled from three sites in Belgium and three in New Zealand, all of which were separated by $>20 \mathrm{~km}$. We randomly sampled and combined five individuals of fourth- or fifth-instar larvae, five workers, and five virgin queens from each of these nests for nucleic acid extraction. When identifiable and available, we also sampled the 'foundress queen' (the wasp queen that founded and initiated the colony the previous spring), which were identified from their characteristically torn wings. Each foundress queen was homogenised for nucleic acid extraction individually. We collected a total of 26 nests from Belgium and 61 from New Zealand over the period 2014-2016 (Appendix 
S1). These samples provided microbial community data and were used in the TaqMan Array Cards (Applied Biosystems, Foster City, California; hereafter referred to as the array).

To assess colony productivity or fitness from each collected nest, the outer nest paper was removed and the combs were laid flat. An image was then captured to estimate the number of worker and queen cells. Worker cells are used to rear workers and the majority of males, whereas the large cells are used for the new queens (Spradbery, 1973). We used IMAGEJ2 software (Rueden et al., 2017) to measure the size of a minimum of 10 large queen cells and 10 small worker cells from photographs of each nest. Then total areas of worker and queen cells were divided by the mean cell size to calculate cell number for each nest. Direct counts of wasps were impossible due to workers foraging and actively defending the nest during the collection process.

We used a permutational MANOVA analysis with 9999 permutations in the ADONIS package (Oksanen et al., 2016) in $\mathrm{R}(\mathrm{R}$ Core Team, 2018) to examine the hypothesis that the relative fitness of wasps was higher in the introduced range. The primary fitness estimator used was the number of queen cells produced per nest, estimated from the number of queen cells in autumn for each nest. Our permutational MANOVA also included the total number of worker cells and the ratio of queen cells to worker cells for each nest.

\section{Network complexity in the introduced and native ranges}

We assessed microbial diversity using nest samples from 2014 in both ranges. We then selected representative prokaryote and eukaryote symbiont/pathogen candidates and mutualists for the array to assess prevalence of selected taxa in individual wasps. The taxa selected for the array included important social insect pathogens and mutualists that are known to be shared among Hymenoptera. For the array we sampled all the nests collected from 2014 to 2016. Codes for the taxa used in the array are in Appendix S1.

Prokaryote microbial diversity was assessed and array targets were chosen based on 16S amplicon sequencing (Quinn, 2017). DNA was extracted using the DNeasy Blood \& Tissue Kit; Qiagen, Hilden, Germany. Amplicons of 16S ribosomal RNA genes were prepared following the Illumina 16S Metagenomic Sequencing Library Preparation Protocol, targeting the V3-V4 region of the 16S rRNA gene using Nextera adapter primers and dual-index barcodes (Illumina, San Diego, California) (Illumina, 2013). Prokaryotes were identified using the QIIME v.1.9.1 pipeline (Caporaso et al., 2010), including PANDASEQ v.2.8 (Masella et al., 2012), the SILVA 128 database (Quast et al., 2013), the PYNAST alignment tool (Caporaso et al., 2010), and CHIMERASLAYER (Haas et al., 2011). Eukaryote microbial diversity and array targets were assessed using $18 \mathrm{~S}$ amplicon sequencing. DNA was extracted using the same method as prokaryote sequencing and amplicons were prepared according to the protocols outlined in the Earth Microbiome Project (Thompson et al., 2017). Eukaryote diversity was analysed using the SILVANGS v.1.3 pipeline (Quast et al., 2013). Viral targets for the array were selected using an Illumina RNAseq approach. The viral candidates were derived from total RNA extracted from wasp samples using the PureLink ${ }^{\circledR}$ RNA Mini Kit and the TruSeq libraries (Thermo Fisher Scientific, Waltham, Massachusetts). RNA was extracted using the methods of Quinn (2017). Viruses were selected based on the methods of Gruber et al. (2017) and Quinn (2017).

After detection and identification of prokaryote, eukaryote and viral candidates, specific array targets were selected in addition to an assay created for the putative immune gene Dscam (Appendix S1). Array assays were designed by the authors and Thermo Fisher Scientific. Samples of individual foundress queens, workers, and gynes from the native and introduced ranges (Belgium, $n=77$, and New Zealand, $n=162$; Appendix S1) were analysed for prevalence of the selected microbial taxa. For each sample, 200 ng cDNA was mixed with TaqMan Fast Advanced Master Mix (Applied Biosystems), and then added to the array cards. The cards were processed as per the manufacturer's instructions and analysed with a QuantStudio 7 Flex Real-Time PCR System (Applied Biosystems; Thermo Fisher Scientific) using recommended PCR conditions. Cycle threshold $(C t)$ values were used to calculate target levels relative to the reference genes using the equation $2^{\left(-C t_{\text {target }}\right)} / 2^{\left(-C t_{\text {reference }}\right)}$.

Relevance networks were derived from Spearman's rank correlations for the $16 \mathrm{~S}, 18 \mathrm{~S}$, and array datasets, split by country and extracted using the TIDYVERSE (Wickham, 2017), CORRR (Jackson, 2016), and IGRAPH (Csardi \& Nepusz, 2006) packages in R. Relevance networks are bipartite graphs where nodes represent variables and edge colour represents the association strength. Only associations exceeding a user-specified threshold are shown in the resulting networks (see Fig. 2 later). Undirected network interactions were visualised in CYTOSCAPE (Shannon et al., 2003), with network statistics collected using NETWORKANALYZER (Assenov et al., 2007).

\section{Is wasp fitness associated with differences in microbial community diversity?}

We hypothesised that the variation in wasp fitness would be associated with differences in the microbial communities. For this analysis we categorised all nests according to five levels of fitness based on the number of queen cells observed in autumn: $<100$, very low; 100-500, low; 500-1000, medium; 1000-1500, high; $>1500$, very high). We fitted permutational MANOVA models with 9999 permutations in the ADONIS package (Oksanen et al., 2016) in R (R Core Team, 2018). The models used Jaccard dissimilarity measures, and initially included interactions between fitness and country, and life stage and country. We then fitted simpler models and chose the best-fitting option using Akaike information criterion values. Separate models were fitted for the $16 \mathrm{~S}, 18 \mathrm{~S}$, and array data. We did not categorise the microbial taxa into mutualists, commensals or pathogens, because very little is known about their influence on wasp biology, and such a classification would be speculative.

\section{Is the expression of the putative immune gene Dscam lower in the introduced range?}

We also included the putative immune gene Dscam in the array. The Dscam assay was designed based on the published 

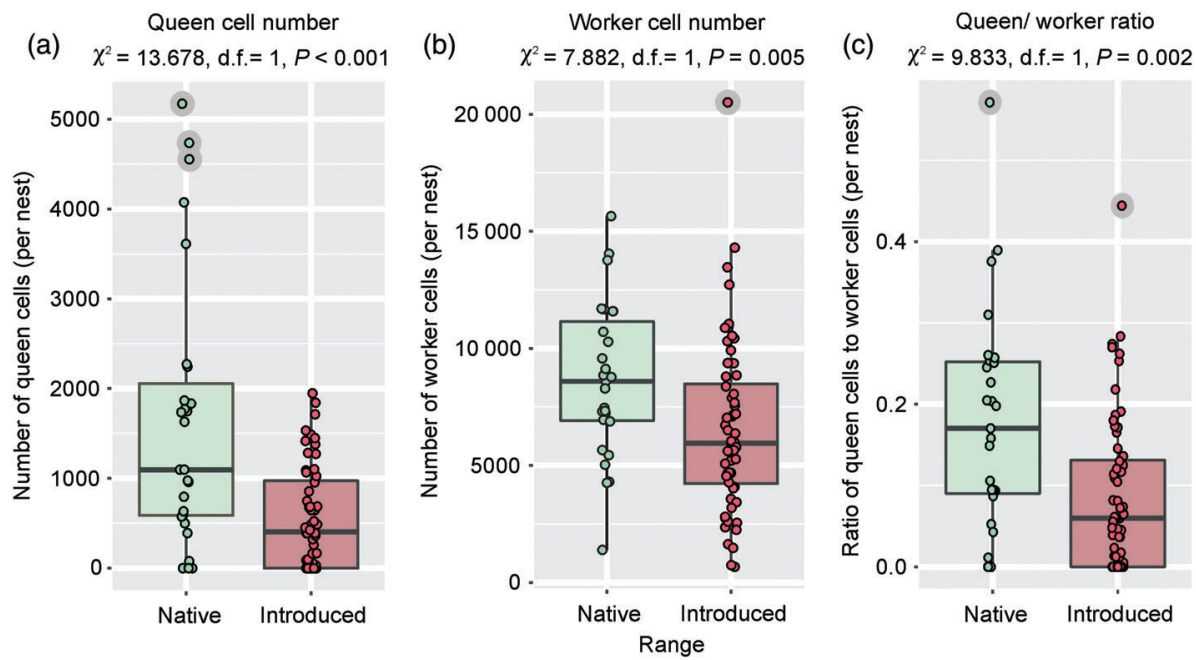

Fig. 1. All estimates of common wasp relative fitness on a per-nest basis were higher in the native (Belgium) than in the introduced (New Zealand) range. $P$-values and $\chi^{2}$ results are from univariate Kruskal-Wallis tests. Boxplots show the mean and the 25th and 75th percentiles, with whiskers extending from the hinge to the highest or lowest value that is within $1.5 \times$ the interquartile range of the hinge, with outliers in grey circles automatically beyond this range. Outliers were not excluded from the associated permutational MANOva. [Colour figure can be viewed at wileyonlinelibrary.com].

Polistes, Bombus, and Apis mellifera genomes, as the Vespula genome was not available. In A. mellifera, the assay region is within terminal exon 1 (GenBank accession AB273636.1). A preliminary analysis indicated the expression patterns were not normally distributed. Consequently, we analysed the data using the 'aovp' function for permutation testing of linear models in the R package LMPERM (Wheeler \& Torchiano, 2016).

\section{Results}

\section{Fitness of wasps in the native and introduced ranges}

The primary fitness estimator that we used was the number of new queen cells produced per nest, which was estimated from the number of queen cells in autumn for each nest. Our permutational MANOvA also included the number of worker cells and the ratio of queen cells to worker cells for each nest. All three fitness measures were correlated (Appendix S1). For all three estimators, fitness was higher in the native than in the introduced range of New Zealand (Fig. 1). The median number of queen cells produced per nest in New Zealand was less than $50 \%$ of the Belgian median (Fig. 1a). This lower queen cell production was correlated with smaller numbers of worker cells in New Zealand nests (Fig. 1b). A permutational MANOVA with the three estimators as response variables and factors of region and year plus a region $\times$ year interaction confirmed significantly higher fitness in the native range $(F=6.745$, d.f. $=1,81, P=0.009)$. Fitness also varied temporally $(F=4.829$, d.f. $=2,81, P=0.008)$, which was expected due to factors that include interannual climate variability (Lester et al., 2017a). There was no significant interaction between region and year $(F=0.477$, d.f. $=2,81$, $P=0.648)$. The median ratio of queen cells to worker cells per nest was nearly three-fold higher in the native range $(0.170)$ than in the introduced range (0.059) (Fig. 1c). There was no evidence for spatial autocorrelation between nests in fitness measures (Appendix S1).

\section{Network complexity in the introduced and native ranges}

We assessed microbial diversity using $16 \mathrm{~S}$ sequencing to examine prokaryotes and $18 \mathrm{~S}$ sequencing for eukaryotes, using nest samples from 2014. Full details of the number of reads and other sequencing results and statistics can be found in Quinn (2017). Our amplicon sequencing identified 467 prokaryote and 80 eukaryote taxa from common wasps. The native range included 358 prokaryote and 38 eukaryote taxa, whereas the introduced range included 386 prokaryote and 61 eukaryote taxa. Of the prokaryote taxa, 277 were present in both New Zealand and Belgium, 109 were absent in the introduced range, and 81 were absent in the native range. Of the 80 eukaryote taxa, 17 were present in both ranges, 19 were absent in the introduced range of New Zealand, and 44 were absent in the Belgian native range.

We examined the hypothesis that the microbial interaction network would be considerably more complex in the native than in the introduced population. Based on the number of taxa and the complexity of each set of networks, we set the correlation threshold for network visualisation and analysis for the $16 \mathrm{~S}$ data to 0.9 , that for the $18 \mathrm{~S}$ data to 0.3 , and that for the array data to 0.15 . Contrary to our expectations, the NETWORKANALYZER results revealed that interaction networks were significantly more complex in the introduced range of New Zealand than in the native range of Belgium for both the $16 \mathrm{~S}$ and $18 \mathrm{~S}$ datasets (Fig. 2). The $16 \mathrm{~S}$ prokaryote network had the most taxa and the highest level of differentiation (Fig. 2a,b). Comparatively, the New Zealand range 16S network showed higher values for the clustering coefficient, number of connected paths, network centralisation, number of shortest paths, mean 
(a) $16 \mathrm{~S}$ - native range (Belgium)

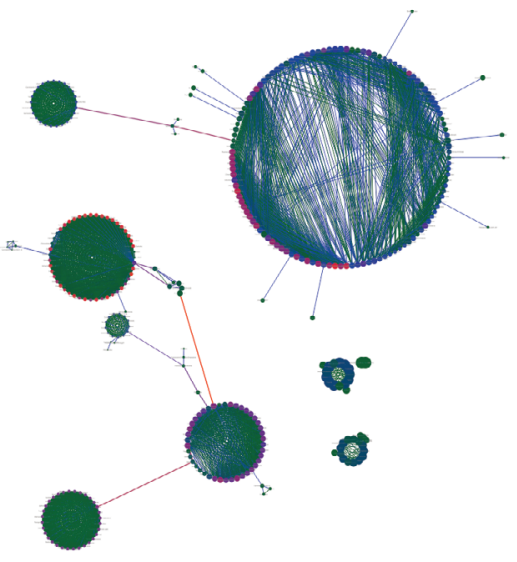

(c) $18 \mathrm{~S}$ - native range (Belgium)

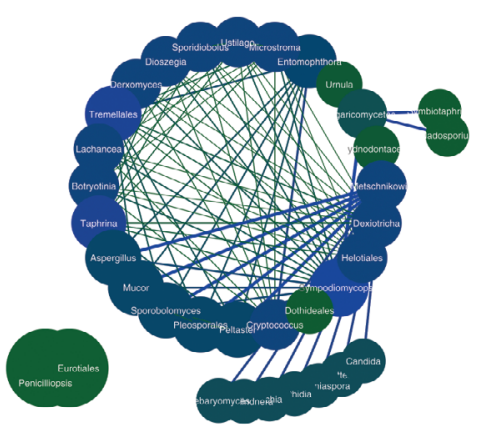

(e) Array- native range (Belgium)

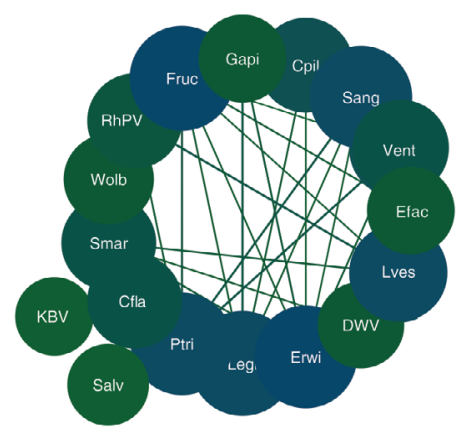

(b) $16 \mathrm{~S}$ - invaded range (New Zealand)

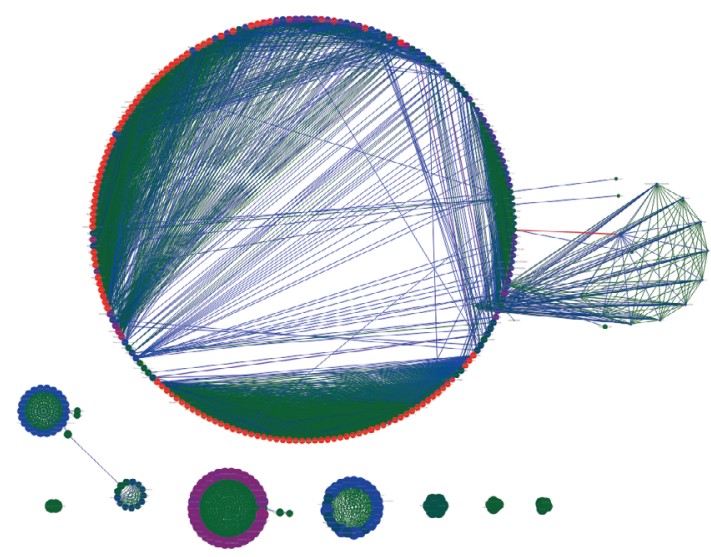

(d) $18 \mathrm{~S}$ - invaded range (New Zealand)

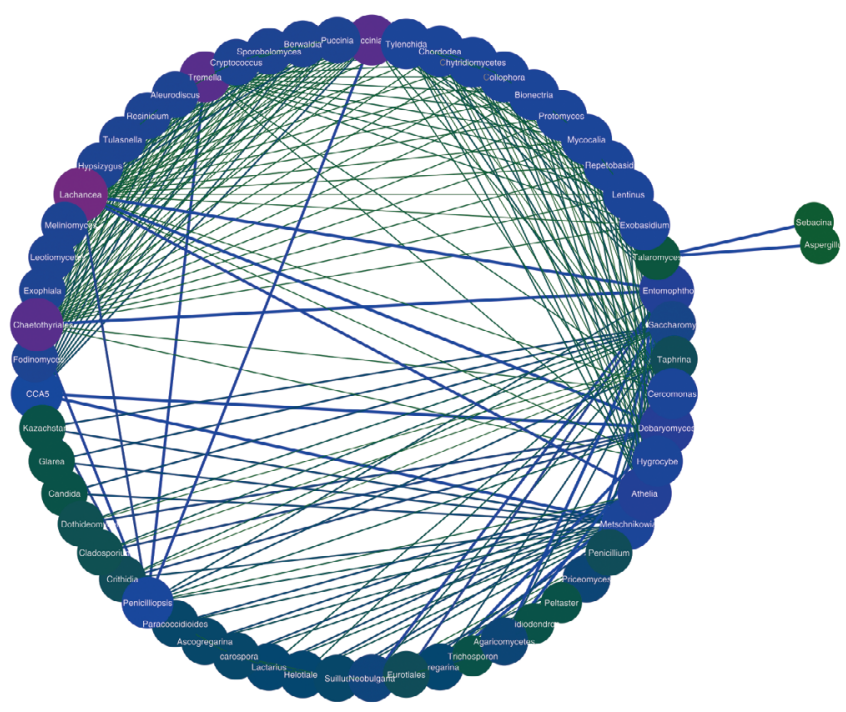

(f) Array - invaded range (New Zealand)

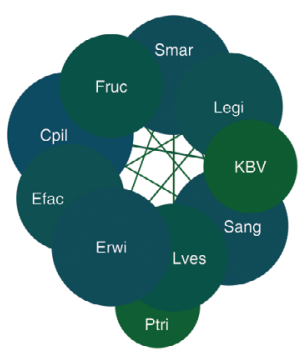

Fig. 2. Hierarchical layouts of undirected $16 \mathrm{~S}$ prokaryote (a, b), $18 \mathrm{~S}$ eukaryote (c, d), and array (e, f) networks of microbial taxa in Belgium and New Zealand. Node properties shown include degree (number of linkages per node) and closeness centrality (average shortest path from one node to all other nodes). Node size is mapped to closeness centrality (low values, smaller circles), and node colour is mapped to degree (low values, green; high values, coral; intermediate values, blue). Edge betweenness (the number of number of shortest paths that pass through an edge) is mapped to edge size and edge colour (low values, thinner lines in green; high values, thicker lines in coral; intermediate values, blue lines). The overall topology comparisons between the networks of the different ranges is most relevant here, with taxa names able to be discerned in figures in the supporting information. 
Table 1. Properties of the microbial networks derived from the $16 \mathrm{~S}, 18 \mathrm{~S}$, and array data in the native (Belgium) and introduced (New Zealand) ranges, derived from NETWORKANALYZER (Assenov et al., 2007) and CYTOSCAPE (Shannon et al., 2003).

\begin{tabular}{|c|c|c|c|c|c|c|}
\hline & \multicolumn{2}{|c|}{ 16S (prokaryotes) } & \multicolumn{2}{|c|}{$18 \mathrm{~S}$ (eukaryotes) } & \multicolumn{2}{|l|}{ Array } \\
\hline & Native & Introduced & Native & Introduced & Native & Introduced \\
\hline Correlation cut-off & 0.90 & 0.90 & 0.30 & 0.30 & 0.15 & 0.15 \\
\hline Clustering coefficient & 0.775 & 0.821 & 0.813 & 0.800 & 0.503 & 0.723 \\
\hline Connected components & 5 & 8 & 2 & 1 & 1 & 2 \\
\hline Network diameter & 17 & 15 & 5 & 4 & 4 & 3 \\
\hline Network radius & 1 & 1 & 1 & 2 & 2 & 1 \\
\hline Network centralisation & 0.065 & 0.107 & 0.318 & 0.296 & 0.263 & 0.286 \\
\hline Shortest paths & 49786 & 57844 & 1058 & 3540 & 272 & 110 \\
\hline Characteristic path length & 5.585 & 4.595 & 2.355 & 2.107 & 1.882 & 1.455 \\
\hline Mean number of neighbours & 20.36 & 27.93 & 8.80 & 14.13 & 5.29 & 4.53 \\
\hline Number of nodes & 349 & 349 & 35 & 60 & 17 & 15 \\
\hline Network density & 0.059 & 0.080 & 0.259 & 0.240 & 0.331 & 0.324 \\
\hline Network heterogeneity & 0.629 & 0.626 & 0.507 & 0.498 & 0.479 & 0.489 \\
\hline Multi-edge node pairs & 3553 & 4874 & 154 & 424 & 45 & 34 \\
\hline
\end{tabular}

The correlation cut-off was chosen for the purpose of meaningful visualisation, based on the size of the network.

Table 2. Results of permutational MANOVA analysis of $16 \mathrm{~S}, 18 \mathrm{~S}$, and array microbial taxa in the native (Belgium) or introduced (New Zealand) range.

\begin{tabular}{|c|c|c|c|c|c|c|}
\hline \multirow[b]{2}{*}{ Predictor term } & \multicolumn{2}{|c|}{ 16S (Prokaryotes) } & \multicolumn{2}{|c|}{$18 \mathrm{~S}$ (Eukaryotes) } & \multicolumn{2}{|l|}{ Array } \\
\hline & $F$-perm & $P$-value & $F$-perm & $P$-value & $F$-perm & $P$-value \\
\hline Range (introduced or native) & 4.980 & $0.001^{* * *}$ & 1.428 & $0.015^{*}$ & 4.297 & $0.003^{* *}$ \\
\hline Fitness & 1.596 & $0.047^{*}$ & 0.964 & 0.605 & 1.166 & 0.258 \\
\hline Life stage & 1.136 & 0.304 & 1.315 & $0.024^{*}$ & 2.105 & $0.005^{* *}$ \\
\hline Year & N/A & N/A & N/A & N/A & 8.227 & $0.001^{* * *}$ \\
\hline Range $\times$ fitness & 1.404 & 0.099 & 0.976 & 0.548 & 1.181 & 0.226 \\
\hline Range $\times$ life stage & 1.111 & 0.285 & 1.313 & $0.019^{*}$ & 2.256 & $0.015^{*}$ \\
\hline Range $\times$ year & N/A & N/A & N/A & N/A & 2.247 & $0.013^{*}$ \\
\hline
\end{tabular}

$* P<0.05$

$* * P<0.01$

$* * * P<0.001$.

The life stages analysed were larvae, adult workers and new queens from each nest. The $16 \mathrm{~S}$ and $18 \mathrm{~S}$ data were only for 2014, whereas the array used a larger number of nests (but fewer microbial taxa) from 2014, 2015 and 2016. N/A, not applicable.

number of neighbours, network density and multi-edge node pairs than were seen in the Belgian native range network (Table 1). The network centralisation parameter is especially informative, as it is frequently interpreted as the risk of a node being influenced (or infected in this case) by whatever is flowing through the network (e.g. one pathogen influencing another). Network complexity was not as well differentiated for the $18 \mathrm{~S}$ eukaryote networks, which naturally had fewer taxa than the $16 \mathrm{~S}$ networks (Fig. 2c,d). However, like the $16 \mathrm{~S}$ network, the $18 \mathrm{~S}$ introduced network had a substantially higher number of shortest paths, mean number of neighbours and multi-edge pairs (Table 1). The array showed less differentiation between the Belgian and New Zealand ranges (Fig. 2e,f), which was expected as there were many fewer taxa in the array. CYTOSCAPE session files for more detail and interactive network visualisation are provided as supporting information (Appendices S2-S8).

\section{Is wasp fitness associated with differences in microbial community diversity?}

The permutational MANOVA analysis of fitness and microbial diversity revealed that microbial communities differed significantly between Belgium and New Zealand for all three datasets $(16 \mathrm{~S}, 18 \mathrm{~S}$, and array). Other factors affected the $16 \mathrm{~S}$, $18 \mathrm{~S}$, and array microbial communities differently (Table 2). Different microbial communities were significantly associated with variation in wasp fitness only for the $16 \mathrm{~S}$ data. Fitness was not significant $(P \geq 0.258)$ for the $18 \mathrm{~S}$ or array data. We also observed statistically significant range $\times$ life stage interactions (18S, $P=0.019$; array, $P=0.015$; Table 2), and range $\times$ year interactions (array, $P=0.013$ ).

Of the 21 microbial taxa screened in the array (Appendix S1), 18 were shared between the ranges, one was absent in New Zealand (Vagococcus entomophilus, a putative commensal), and two were absent in Belgium (Acetobacter and Bifidobacterium, both putative commensals). Differences in the microbiome between countries and life stages were highlighted by viral infection results from the array data (Fig. 3). The three viruses examined on the array were found in both ranges. The Kashmir bee virus $(\mathrm{KBV})$ was the most commonly observed virus, in 147 of the 278 samples analysed, and in larvae, worker, and queens from both the native and introduced ranges. The KBV infection rate of $65 \%$ of all samples observed in New Zealand was nearly three-fold and significantly higher than the $23 \%$ in 
(a) Kashmir bee virus (KBV)
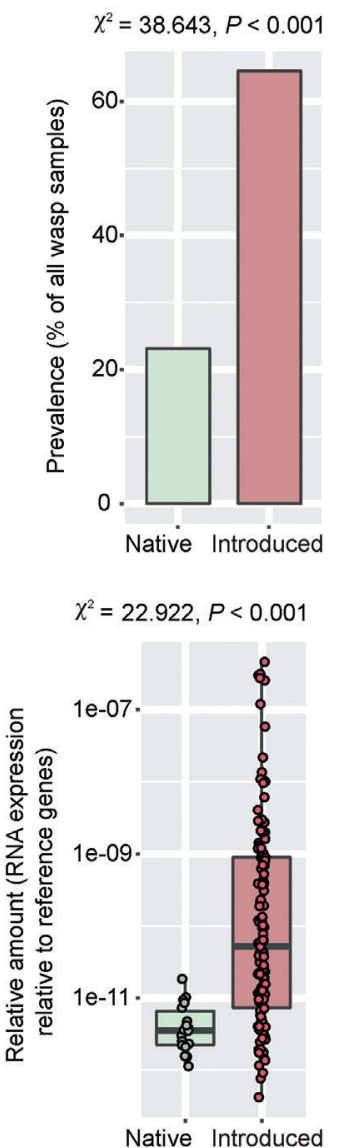

(b) (DWV)

$\chi^{2}=6.103, P=0.014$

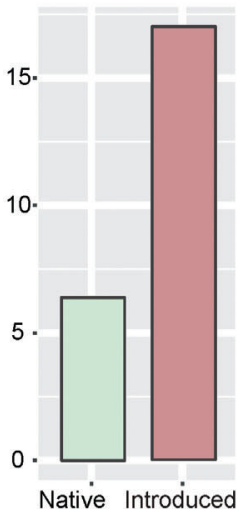

Native Introduced

$\chi^{2}=0.706, P<0.401$

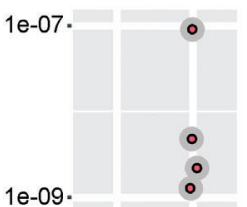

$1 \mathrm{e}-09$.

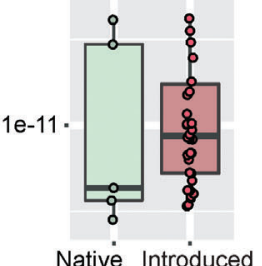

(c)

Rhopalosiphum aphid virus (RhPV)

$\chi^{2}=53.762, P<0.001$

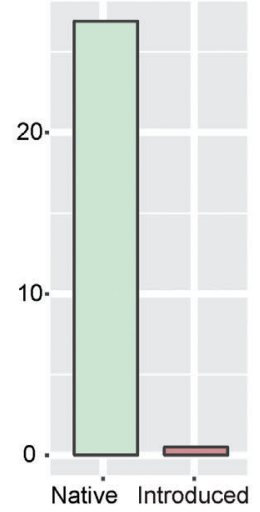

Native Introduced

$\chi^{2}=0.503, P<0.478$

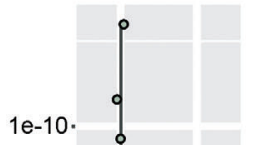

$1 e-10$
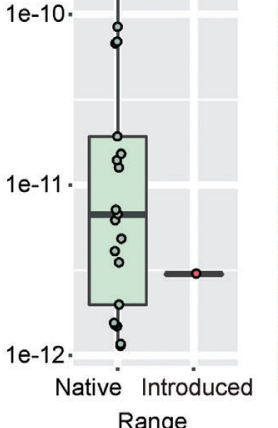

(d) Aspergillus

$\chi^{2}=10.658, P=0.001$

15 .

0 .

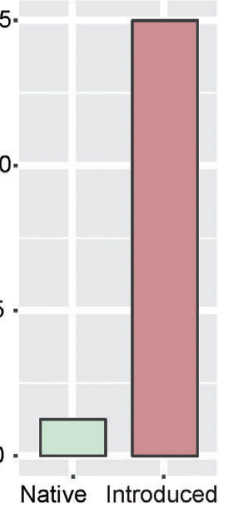

$\chi^{2}=1.513, P=0.219$

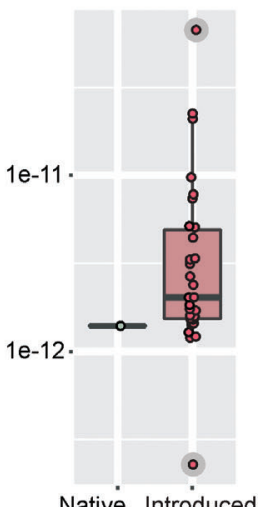

(e)

Lactobacillus

$\chi^{2}=1.363, P=0.243$

60 .

40

Native Introduced

$\chi^{2}=0.206, P=0.650$

$1 \mathrm{e}-09$.

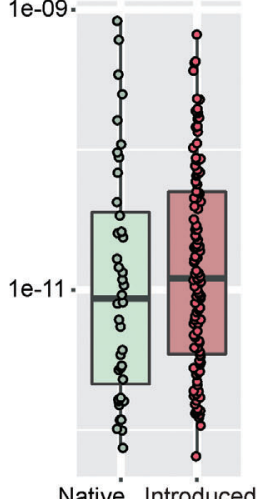

Fig. 3. The prevalence (upper graphs) and relative abundance (lower graphs) of five microbial taxa from the TaqMan array analysis. The prevalence was based on the total number of wasp samples, from all life stages, in which the microbe was observed in each range. $P$-values and $\chi^{2}$ results at the top of prevalence graphs are from two-sample tests for independent proportions. The relative abundance is based on the RNA expression for each target relative to the reference gene. $P$-values and $\chi^{2}$ results are from univariate Kruskal-Wallis tests. [Colour figure can be viewed at wileyonlinelibrary.com].

Belgium. KBV was also present in significantly higher titres in the introduced range (Fig. 3a). The Deformed wing virus (DWV) was also nearly three-fold and significantly more prevalent in the introduced range of New Zealand than in the Belgian native range (Fig. 3b). The DWV was not observed in queens from either range, often despite workers in the same nest being infected. The Rhopalosiphum padi virus (RhPV) was observed in $27 \%$ of samples from the native range and in all life stages there (Fig. 3c). However, RhPV was found at extremely low titres from only one sample in New Zealand, which could have been a false-positive.

Of the prokaryote taxa on the array, like the viruses, some putative pathogens such as Aspergillus were more prevalent in the introduced range (15\% vs. 1\%; Fig. 3d). Others, such as the Lotmaria/Crithidia target, had a similar rate of infection between ranges (12\% in the introduced vs. $10 \%$ in the native; $\chi^{2}=0.088$, d.f. $\left.=1, P=0.767\right)$. Our assay for the putative target Enterococcus faecalis (a Gram-positive, commensal bacterium previously found in the gastrointestinal tracts of humans and other mammals) showed a remarkably similar prevalence between ranges, at $64 \%$ and $65 \%$ of samples in the introduced and native range, respectively, with a median abundance almost exactly the same in the two ranges. The likely mutualistic bacterial genus Lactobacillus also showed similar rates of prevalence and abundance in both ranges (Fig. 3e).

\section{Is the expression of the putative immune gene Dscam lower in the introduced range?}

Wasps in the introduced range of New Zealand always showed higher median expression of the putative immune gene Dscam than in the Belgian native range $(P=0.002$; Fig. 4; Appendix S1). Different wasp life stages also showed varying expression patterns $(P<0.001$; Appendix S1), with the lowest median values for larvae in the native range and the highest for adult worker wasps in the introduced range. The variation between sampling years in Dscam expression $(P<0.001$; Appendix S1) 


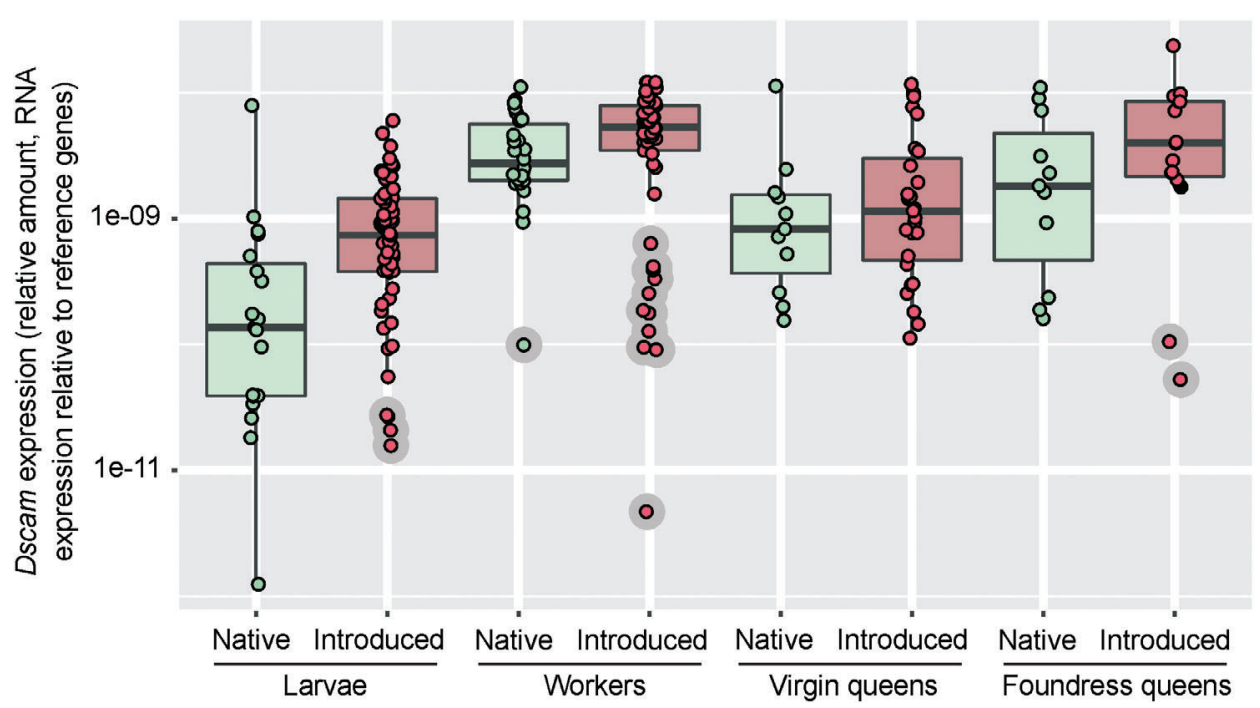

Fig. 4. The relative expression of the immune gene Down syndrome cell adhesion molecule (Dscam) from life stages of wasps in both the native (Belgium) and introduced (New Zealand) ranges, from the TaqMan array analysis. Foundress queens refer to the queen wasp that had been present in the nest and produced all the larvae, workers, and virgin queens in autumn. Median expression levels of Dscam within each life stage were always higher in the introduced range, relative to the native. The relative expression is based on the mRNA quantification for Dscam relative to the reference gene. [Colour figure can be viewed at wileyonlinelibrary.com].

is consistent with the significant variation between years in the relative abundance of microbial taxa from the array data (Table 2).

\section{Discussion}

Wasp nest fitness in the introduced range was approximately half that of the native range. The median number of queen cells per nest in the native range in Belgium (1097) was over twice as high as in the introduced range in New Zealand (404). Further, these results are likely to be compounded by the previously observed relationship between nest size and queen quality. Harris and Beggs (1995) found that smaller nests typically produce smaller queens, and smaller queens are under-represented in the reproductive population of the following year. Our results also indicate that it takes $c .16 .9$ workers to rear a queen in the introduced range, which is nearly three-fold higher than in the native range (5.9 workers). This result contrasts with conclusions from a meta-analysis of 53 species by Parker et al. (2013), who found strong evidence for a typically enhanced reproductive output and organism size in the introduced relative to the native range. Only a single species had a relatively reduced reproductive output in the introduced range (an amphipod shrimp, Crangonyx pseudogracilis) (Parker et al., 2013). Our study organism, the common wasp, was cited in this review as having higher abundance in the introduced range, but no data for reproductive output were presented (Parker et al., 2013). Indeed, on a landscape scale, these wasps show a contrasting pattern of abundance and relative fitness to our finer-scale analysis. The median number of nests from long-term surveys in this area of New Zealand is 11.8 nests ha ${ }^{-1}$ (Lester et al., 2017a) compared with 0.45 nests ha ${ }^{-1}$ in England (Archer \& Halstead, 2014). Simple multiplication of median queen cells per nest and nest density indicates that at a landscape-scale queen production in the introduced range is approximately 10 -fold higher each year than in the native range, which is the opposite of our finding for individual nests. The relatively high landscape-level nest density is likely to be a combination of many factors, including food and resource availability, as well as a lack of any other competing Vespula or social wasp species (Lester \& Beggs, 2019). There is likely to be much more intraspecific competition in New Zealand than in Belgium. This high landscape-level nest density and wasp abundance have implications for the spillover and spillback of pathogens and parasites, and the enemy release hypothesis.

Different microbial communities formed in each of the host ranges. The enemy release hypothesis is one of the most frequently cited explanations for invasion success, which relies on the key condition that 'enemy loss' occurs, and the assumption that these enemies have substantial negative effects on the invasive species' performance in their native range (Prior et al., 2014). In the native range, our analysis found 358 prokaryote and 38 eukaryote taxa, and the introduced range included 386 prokaryote and 61 eukaryote taxa. These richness estimates fall between estimates for the microbial diversity in bees and invasive ants using similar methods (Hubert et al., 2015; Lester et al., 2017b). Our permutational MANOva used a presence or absence matrix and indicated significant effects of the prokaryote community on wasp fitness (Table 2). This analysis is suggestive that variation in microbial communities is associated with the relative fitness of these invasive wasps, as has been shown in honey bees (Bromenshenk et al., 2010; Evans \& Schwarz, 2011; Cornman et al., 2012). There is evidence for the hologenome paradigm in honey bees, which suggests that their fitness is determined by a composite of bee and microbiota genomes (Schwarz et al., 2015), which appears also to 
be likely for the common wasp. We have previously found fitness effects of viruses on wasps in New Zealand (Dobelmann et al., 2017). Our current study is the first to find an effect on fitness of prokaryote communities. It is possible that the bacteria we detected are candidates for biological control of wasps, although our results could also be associated with microbial species that are missing from the introduced range of New Zealand. Prokaryote communities were also significantly different between ranges. This result could indicate a degree of enemy or mutualist loss, although the description and role of many of the taxa involved here are lacking, because of the paucity of research on the function of the microbial fauna of wasps.

Many of the microbial taxa were observed in both the introduced and native ranges. Our previous work has similarly found no evidence of microbial enemy loss for common wasp populations in New Zealand or elsewhere in their introduced range (Lester et al., 2015), despite evidence of as few as six successful introductions for the New Zealand populations (Lester et al., 2014). Putative pathogens such as Nosema spp. and Actinobacteria are present throughout the wasps' distribution (Lester et al., 2015). Similar results showing a lack of enemy release despite a genetic bottleneck have been shown for several introduced species, including the German wasp Vespula germanica (Brenton-Rule et al., 2018), starlings (Colautti et al., 2005) and freshwater amphipods (Slothouber Galbreath et al., 2009). Lack of enemy release for starlings is thought to be due to a combination of the birds bringing parasites with them, acquiring parasites from their native range through alternative hosts, and acquiring parasites from birds in the introduced range (Colautti et al., 2005). This pattern has probably been repeated with common wasps.

Our analysis also indicated differences between ranges that included the apparent loss of some taxa and acquisition of others that are likely to be pathogens. The array analysis comprised the greatest number of samples and provided the highest confidence for the probable absence of species such as RhPV from wasps in New Zealand. The RhPV virus was initially described from aphids but has since been observed in honey bees and a parasitic wasp (Oliveira et al., 2010; Roberts et al., 2018). Common wasps in the introduced range appear to have acquired Aspergillus, which is a pathogen known from honey bees (Foley et al., 2014) and previously from wasps in New Zealand (Glare et al., 1996). Many of the viruses, bacterial and fungal pathogens identified in our work are generalist taxa that have been observed in a range of arthropod hosts (e.g. Levitt et al., 2013; Gruber et al., 2017; Loope et al., 2019). Like most other countries, New Zealand has had a range of exotic insect introductions, including honey bees, 12 other bee species, paper and German wasps, and many parasitoids introduced for biological control purposes. Pathogen spillover and spillback from such species are considered likely in invasive species introductions and there is evidence of this in hymenopteran communities (Singh et al., 2010; Fürst et al., 2014; Faillace et al., 2017; Loope et al., 2019). Similarly, in other ecosystems such as grasslands, the disease prevalence can be explained by the abundance of the focal host and the abundance of all related species in the community that may share the disease
(Parker et al., 2015). Consequently, although a small number of introduction events might limit the pathogens brought by any one introduced population into a new community and range, prior introductions from a wide variety of related exotic species might have already populated the landscape with a rich and diverse microbial community containing many generalist pathogens.

Epidemiological theory predicts that a high density of pathogen and microbial hosts would result in a high rate of disease infection and prevalence, and highly connected microbial networks. Within social insect nests, increasing colony size and complex interaction networks are predicted to facilitate pathogen transmission and spread (Naug \& Camazine, 2002). Importantly, intercolony exchange of workers has frequently been observed in wasps, with estimates ranging from 1-2\% of workers in Vespula maculifrons (Lord et al., 1977) to $56 \%$ of workers in Polistes canadensis moving among nests situated within overlapping foraging ranges (Sumner et al., 2007). This exchange of workers between nests combined with the potential acquisition of pathogens via foraging in a common environment (Singh et al., 2010) would facilitate a highly connected pathogen and microbial network. Pathogen infection risk is typically associated with high connectivity via individuals or the common use of habitat (Godfrey, 2013; White et al., 2017). Consequently, it was not surprising that the high nest densities observed in the introduced range (Lester et al., 2017a) have led to relatively dense, centralised microbial networks. The prokaryote interaction network in the introduced range was especially complex, showing higher values for the clustering coefficient, network centralisation, number of shortest paths, mean number of neighbours, and network density. It was not surprising either that the lower nest densities and likely low rate of contact and exchange of worker wasps in the native range could have the opposite effect.

The expression of the putative immune gene Dscam was significantly higher in the introduced range, which was another result that was inconsistent with our predictions. The median Dscam expression in worker wasps from the native range was only $52 \%$ of that in the introduced range. Although Dscam is known to be up-regulated by viral challenges in honey bees (Brutscher et al., 2015) and is thought to have a broad role in invertebrate immune systems ( $\mathrm{Ng}$ et al., 2014), its precise function in wasps is unknown. The patterns of Dscam expression might instead suggest that wasps face a greater challenge from pathogens and parasites in the introduced range of New Zealand than in the Belgium native range. That result is consistent with our array data and the network analyses, as well as the observation that it takes approximately three-fold more workers to produce each queen in the introduced than it does in the native range. Little is known, however, about Dscam in these wasps, and experimental manipulation would be needed to confirm this result for this species.

On an individual nest basis, our results do not support the hypothesis that $V$. vulgaris performs better in their New Zealand introduced range (Parker et al., 2013). On a landscape scale in this area of the introduced range, however, these wasps are managing to attain the world's highest known densities for this species. 


\section{Acknowledgements}

We thank the many field assistants involved in wasp nest collection. We thank two referees for comments that substantially improved this manuscript. This work was supported by the Ministry of Business, Innovation and Employment (New Zealand's Biological Heritage NSC, C09X1501. It was also supported by a Royal Society Te Apārangi Marsden grant to PJL, MAMG, and $\mathrm{JH}$.

\section{Author contributions}

PJL and MAMG conceived the study. PJL, MAMG, and OQ designed the study. OQ collected the data with the support of JWB, JD, and TW. OQ, MAMG, PJL, and JH analysed the data. MAMG and PJL led the writing of the manuscript with contributions from all authors. The authors declare that they have no conflicts of interest.

\section{Supporting Information}

Additional supporting information may be found online in the Supporting Information section at the end of the article.

Appendix S1. Supplementary methods showing sampling locations and sample sizes, primers for microbial taxa, correlations between nest productivity parameters, spatial autocorrelation analyses, a description of the CYTOSCAPE session files, and the permutational ANOVA on the putative immune gene Dscam expression data.

Appendix S2. network.17052018.cys is the CYTOSCAPE session file with all six networks for visualisation. CYTOSCAPE should be installed, and then the file can be opened.

Appendix S3. network.16s.be.graphml is the graph file for the 16S Belgium data. This can be opened with any graph analysis package (and can be imported into CYTOSCAPE for visualisation).

Appendix S4. network.16s.nz.graphml is the graph file for the $16 \mathrm{~S}$ New Zealand data. This can be opened with any graph analysis package (and can be imported into CYTOSCAPE for visualisation).

Appendix S5. network.18s.be.graphml is the graph file for the $18 \mathrm{~S}$ Belgium data. This can be opened with any graph analysis package (and can be imported in to CYTOSCAPE for visualisation).

Appendix S6. network.18s.nz.graphml is the graph file for the $18 \mathrm{~S}$ New Zealand data. This can be opened with any graph analysis package (and can be imported in to CYTOSCAPE for visualisation).

Appendix S7. network.array.be.graphml is the graph file for the Belgium array data. This can be opened with any graph analysis package (and can be imported in to CYTOSCAPE for visualisation).

Appendix S8. network.array.nz.graphml is the graph file for the New Zealand array data. This can be opened with any graph analysis package (and can be imported in to CYTOSCAPE for visualisation).

\section{References}

Archer, M.E. \& Halstead, A. (2014) Population dynamics of social wasps (Hymenoptera: Vespidae) in the Royal Horticultural Society's garden at Wisley, Surrey. Entomologist's Monthly Magazine, 150, $19-26$.

Armitage, S.A.O., Peuß, R. \& Kurtz, J. (2015) Dscam and pancrustacean immune memory - a review of the evidence. Developmental and Comparative Immunology, 48, 315-323.

Assenov, Y., Ramírez, F., Schelhorn, S.-E., Lengauer, T. \& Albrecht, M. (2007) Computing topological parameters of biological networks. Bioinformatics, 24, 282-284.

Brenton-Rule, E.C., Dobelmann, J., Brown, B., Dvořák, L., Massiochi, M., Shortall, C.R. et al. (2018) The origins of global invasions of the German wasp (Vespula germanica) and its infection with two honey bee viruses. Diversity and Distributions, 24, 1-1.

Bromenshenk, J.J., Henderson, C.B., Wick, C.H., Stanford, M.F., Zulich, A.W., Jabbour, R.E. et al. (2010) Iridovirus and microsporidian linked to honey bee colony decline. PLoS One, $\mathbf{5}$, e13181.

Brutscher, L.M., Daughenbaugh, K.F. \& Flenniken, M.L. (2015) Antiviral defense mechanisms in honey bees. Current Opinion in Insect Science, 10, 71-82.

Caporaso, J.G., Kuczynski, J., Stombaugh, J., Bittinger, K., Bushman, F.D., Costello, E.K. et al. (2010) QIIME allows analysis of high-throughput community sequencing data. Nature Methods, 7 , $335-336$.

Colautti, R.I., Muirhead, J.R., Biswas, R.N. \& MacIsaac, H.J. (2005) Realized vs apparent reduction in enemies of the European starling. Biological Invasions, 7, 723-732.

Cornman, R.S., Tarpy, D.R., Chen, Y., Jeffreys, L., Lopez, D., Pettis, J.S. et al. (2012) Pathogen webs in collapsing honey bee colonies. PLoS One, 7, e43562.

Csardi, G. \& Nepusz, T. (2006) The igraph software package for complex network research. InterJournal Complex Systems, 1695, $1-9$.

Dobelmann, J., Loope, K.J., Wilson-Rankin, E., Quinn, O., Baty, J.W., Gruber, M.A.M. et al. (2017) Fitness in invasive social wasps: the role of variation in viral load, immune response and paternity in predicting nest size and reproductive output. Oikos, 126, 1208-1218.

Evans, J.D. \& Schwarz, R.S. (2011) Bees brought to their knees: microbes affecting honey bee health. Trends in Microbiology, 19, 614-620.

Evison, S.E.F., Roberts, K.E., Laurenson, L., Pietravalle, S., Hui, J., Biesmeijer, J.C. et al. (2012) Pervasiveness of parasites in pollinators. PLoS One, 7, e30641.

Faillace, C.A., Lorusso, N.S. \& Duffy, S. (2017) Overlooking the smallest matter: viruses impact biological invasions. Ecology Letters, 20, 524-538.

Flenniken, M.L. \& Andino, R. (2013) Non-specific dsRNA-mediated antiviral response in the honey bee. PLoS One, 8, e77263.

Foley, K., Fazio, G., Jensen, A.B. \& Hughes, W.O.H. (2014) The distribution of Aspergillus spp. opportunistic parasites in hives and their pathogenicity to honey bees. Veterinary Microbiology, 169, 203-210.

Fürst, M.A., McMahon, D.P., Osborne, J.L., Paxton, R.J. \& Brown, M.J.F. (2014) Disease associations between honeybees and bumblebees as a threat to wild pollinators. Nature, 506, 364-366.

Glare, T.R., Harris, R.J. \& Donovan, B.J. (1996) Aspergillus flavus as a pathogen of wasps, Vespula spp., in New Zealand. New Zealand Journal of Zoology, 23, 339-344. 
Godfrey, S.S. (2013) Networks and the ecology of parasite transmission: a framework for wildlife parasitology. International Journal for Parasitology: Parasites and Wildlife, 2, 235-245.

Gruber, M.A.M., Cooling, M., Baty, J.W., Buckley, K., Friedlander, A., Quinn, O. et al. (2017) Single-stranded RNA viruses infecting the invasive Argentine ant, Linepithema humile. Scientific Reports, 7, 3304

Haas, B.J., Gevers, D., Earl, A.M., Feldgarden, M., Ward, D.V., Giannoukos, G. et al. (2011) Chimeric 16S rRNA sequence formation and detection in sanger and 454-pyrosequenced PCR amplicons. Genome Research, 21, 494-504.

Harris, R.J. \& Beggs, J.R. (1995) Variation in the quality of Vespula vulgaris (L.) queens (Hymenoptera: Vespidae) and its significance in wasp population dynamics. New Zealand Journal of Zoology, 22, 131-142.

Hubert, J., Erban, T., Kamler, M., Kopecky, J., Nesvorna, M., Hejdankova, S. et al. (2015) Bacteria detected in the honeybee parasitic mite Varroa destructor collected from beehive winter debris. Journal of Applied Microbiology, 119, 640-654.

Illumina. (2013) 16S Metagenomic sequencing library preparation. [WWW document]. URL https://support.illumina.com/documents/ documentation/chemistry_documentation/16s/16s-metagenomiclibrary-prep-guide-15044223-b.pdf [accessed on 1 March 2019].

Jackson, S. (2016) corrr: Correlations in R. R package version 0.2.1. https://CRAN.R-project.org/package=corrr.

Kaltenpoth, M. \& Engl, T. (2014) Defensive microbial symbionts in Hymenoptera. Functional Ecology, 28, 315-327.

Keane, R.M. \& Crawley, M.J. (2002) Exotic plant invasions and the enemy release hypothesis. Trends in Ecology \& Evolution, 17, $164-170$.

Lester, P.J. \& Beggs, J.R. (2019) Invasion success and management strategies for social Vespula wasps. Annual Review of Entomology, 64, 51-71.

Lester, P.J., Bosch, P.J., Gruber, M.A.M., Kapp, E.A., Peng, L., Brenton-Rule, E.C. et al. (2015) No evidence of enemy release in pathogen and microbial communities of common wasps (Vespula vulgaris) in their native and introduced range. PLoS One, 10, e0121358.

Lester, P.J., Gruber, M.A.M., Brenton-Rule, E.C., Archer, M.E., Corley, J.C., Dvorak, L. et al. (2014) Determining the origin of invasions and demonstrating a lack of enemy release from microsporidian pathogens in common wasps (Vespula vulgaris). Diversity and Distributions, 20, 964-974.

Lester, P.J., Haywood, J., Archer, M.E. \& Shortall, C.R. (2017a) The long-term population dynamics of common wasps in their native and invaded range. Journal of Animal Ecology, 86, 337-347.

Lester, P.J., Sebastien, A., Suarez, A.V., Barbieri, R.F. \& Gruber, M.A.M. (2017b) Symbiotic bacterial communities in ants are modified by invasion pathway bottlenecks and alter host behavior. Ecology, 98, 861-874.

Levitt, A.L., Singh, R., Cox-Foster, D.L., Rajotte, E., Hoover, K., Ostiguy, N. et al. (2013) Cross-species transmission of honey bee viruses in associated arthropods. Virus Research, 176, 232-240.

Loope, K.J., Baty, J.W., Lester, P.J. \& Wilson Rankin, E.E. (2019) Pathogen shifts in a honeybee predator following the arrival of the Varroa mite. Proceedings of the Royal Society Series B, 286, 20182499.

Lord, W.D., Nicolson, D.A. \& Roth, R.R. (1977) Foraging behavior and colony drift in Vespula maculifrons (Buysson) (Hymenoptera: Vespidae). Journal of the New York Entomological Society, 85, 186.

Lowe, S., Browne, M., Boudjelas, S. \& De Poorter, M. (2000) 100 of the World's Worst Invasive Alien Species. A Selection from the Global Invasive Species Database. The Invasive Species Specialist Group
(ISSG) a Specialist Group of the Species Survival Commission (SSC) of the World Conservation Union. IUCN, Gland, Switzerland.

Masella, A.P., Bartram, A.K., Truszkowski, J.M., Brown, D.G. \& Neufeld, J.D. (2012) PANDAseq: paired-end assembler for illumina sequences. BMC Bioinformatics, 13, 31 .

McFall-Ngai, M., Hadfield, M.G., Bosch, T.C.G., Carey, H.V., Domazet-Lošo, T., Douglas, A.E. et al. (2013) Animals in a bacterial world, a new imperative for the life sciences. Proceedings of the National Academy of Sciences, 110, 3229-3236.

Naug, D. \& Camazine, S. (2002) The role of colony organization on pathogen transmission in social insects. Journal of Theoretical Biology, 215, 427-439.

Ng, T.H., Chiang, Y.-A., Yeh, Y.-C. \& Wang, H.-C. (2014) Review of Dscam-mediated immunity in shrimp and other arthropods. Developmental and Comparative Immunology, 46, 129-138.

Oksanen, J., Blanchet, F.G., Friendly, M., Kindt, R., Legendre, P. \& McGlinn, D., et al. (2016) vegan: Community Ecology Package. R package version 2.4-0. https://CRAN.R-project.org/package=vegan.

Oliveira, D.C.S.G., Hunter, W.B., Ng, J., Desjardins, C.A., Dang, P.M. \& Werren, J.H. (2010) Data mining cDNAs reveals three new single stranded RNA viruses in Nasonia (hymenoptera: Pteromalidae) Insect Molecular Biology, 19, 99-107.

Parker, I.M., Saunders, M., Bontrager, M., Weitz, A.P., Hendricks, R., Magarey, R. et al. (2015) Phylogenetic structure and host abundance drive disease pressure in communities. Nature, 520, 542-544.

Parker, J.D., Torchin, M.E., Hufbauer, R.A., Lemoine, N.P., Alba, C., Blumenthal, D.M. et al. (2013) Do invasive species perform better in their new ranges? Ecology, 94, 985-994.

Prior, K.M., Powell, T.H.Q., Joseph, A.L. \& Hellmann, J.J. (2014) Insights from community ecology into the role of enemy release in causing invasion success: the importance of native enemy effects. Biological Invasions, 17, 1283-1297.

Quast, C., Pruesse, E., Yilmaz, P., Gerken, J., Schweer, T., Yarza, P. et al. (2013) The SILVA ribosomal RNA gene database project: improved data processing and web-based tools. Nucleic Acids Research, 41, D590-D596.

Quinn, O. (2017) Microbiota of an invasive wasp Vespula vulgaris and Hymenopteran relatives. Unpublished $\mathrm{PhD}$ thesis, Victoria University of Wellington.

R Core Team (2018) R: a language and environment for statistical computing. R Foundation for Statistical Computing, Vienna. https:// www.R-project.org/. https://www.R-project.org.

Roberts, J.M.K., Anderson, D.L. \& Durr, P.A. (2018) Metagenomic analysis of Varroa-free Australian honey bees (Apis mellifera) shows a diverse Picornavirales virome. Journal of General Virology, 336, 1304-1309.

Rose, E.A.F., Harris, R.J. \& Glare, T.R. (1999) Possible pathogens of social wasps (Hymenoptera: Vespidae) and their potential as biological control agents. New Zealand Journal of Zoology, 26, $179-190$

Rueden, C.T., Schindelin, J., Hiner, M.C., DeZonia, B.E., Walter, A.E., Arena, E.T. et al. (2017) ImageJ2: ImageJ for the next generation of scientific image data. BMC Bioinformatics, 18, 529.

Schmucker, D., Clemens, J.C., Shu, H., Worby, C.A., Xiao, J., Muda, M. et al. (2000) Drosophila Dscam is an axon guidance receptor exhibiting extraordinary molecular diversity. Cell, 101, 671-684.

Schwarz, R.S. \& Evans, J.D. (2013) Single and mixed-species trypanosome and microsporidia infections elicit distinct, ephemeral cellular and humoral immune responses in honey bees. Developmental and Comparative Immunology, 40, 300-310.

Schwarz, R.S., Huang, Q. \& Evans, J.D. (2015) Hologenome theory and the honey bee pathosphere. Current Opinion in Insect Science, 10, $1-7$. 
Shannon, P., Markiel, A., Ozier, O., Baliga, N.S., Wang, J.T., Ramage, D. et al. (2003) Cytoscape: a software environment for integrated models of biomolecular interaction networks. Genome Research, 13, $2498-2504$.

Shapira, M. (2016) Gut microbiotas and host evolution: scaling up symbiosis. Trends in Ecology \& Evolution, 31, 539-549.

Singh, R., Levitt, A.L., Rajotte, E., Holmes, E.C., Ostiguy, N., vanEngelsdorp, D. et al. (2010) RNA viruses in hymenopteran pollinators: evidence of inter-taxa virus transmission via pollen and potential impact on non-Apis hymenopteran species. PLoS One, $\mathbf{5}$, e14357.

Slothouber Galbreath, J.G.M., Smith, J.E., Becnel, J.J., Butlin, R.K. \& Dunn, A.M. (2009) Reduction in post-invasion genetic diversity in Crangonyx pseudogracilis (Amphipoda: Crustacea): a genetic bottleneck or the work of hitchhiking vertically transmitted microparasites? Biological Invasions, 12, 191-209.

Spradbery, J.P. (1971) Seasonal changes in the population structure of wasp colonies (Hymenoptera: Vespidae). Journal of Animal Ecology, 40, 501-523.

Spradbery, J.P. (1973) Wasps: An Account of the Biology and Natural History of Solitary and Social Wasps. University of Washington Press, Seattle, Washington.
Sumner, S., Lucas, E., Barker, J. \& Isaac, N. (2007) Radio-tagging technology reveals extreme nest-drifting behavior in a eusocial insect. Current Biology, 17, 140-145.

Thompson, L.R., Sanders, J.G., McDonald, D., Amir, A., Ladau, J., Locey, K.J. et al. (2017) A communal catalogue reveals Earth's multiscale microbial diversity. Nature, 551, 457-463.

Torchin, M.E., Lafferty, K.D., Dobson, A.P., McKenzie, V.J. \& Kuris, A.M. (2003) Introduced species and their missing parasites. Nature, 421, 628-630.

Wheeler, B. \& Torchiano, M. (2016) ImPerm: Permutation Tests for Linear Models. R package version 2.1.0. http://CRAN.R-project.org/ package $=1 \mathrm{mPerm}$.

White, L.A., Forester, J.D. \& Craft, M.E. (2017) Dynamic, spatial models of parasite transmission in wildlife: their structure, applications and remaining challenges. Journal of Animal Ecology, 87, 559-580.

Wickham, H. (2017) tidyverse: Easily Install and Load "Tidyverse." $\mathrm{R}$ package version 1.2.1. http://CRAN.R-project.org/ package $=$ tidyverse.

Accepted 25 January 2019

First published online 9 March 2019

Associate Editor: Sheena Cotter 